\title{
Ueber die Integration der hydrodynamischen Gleichungen.
}

(Von A. Clebsch zu Carlsruhe.)

$\$ 1$.
In einem frühern Aufsatze (dieses Journal, Bd. 54, p. 254) habe ich ein Theorem entwickelt, welches die Integration der hydrodynamischen Gleichungen für die stationäre Bewegung zurückführt auf ein System von zwei partiellen Differentialgleichungen zweiter Ordnung; oder auf die Aufgabe, ein gewisses Integral zu einem Minimum zu machen, bei welchem die zu integrirende Function die lebendige Kraft darstellt. Dies wurde erreicht, indem die Geschwindigkeiten durch zwei neue Functionen ausgedrückt wurden, welche Constanten gleich gesetzt, Integrale der hinzutretenden gewöhnlichen Differentialgleichungen waren, und die Continuitälsgleichung identisch erfüllten. Die Ausdehnung dieses Verfahrens auf den Fall der nic's' stationären Bewegung führte zu sehr verwickelten Gleichungen, welche keine Zurückführung auf ein Problem der Variationsrechnung gestatteten.

Indefs habe ich gefunden, dafs auch dieser allgemeine Fall immer auf ein solches Problem zurückgeführt werden kann, und zwar auf das Inlegral einer Function, welche sich von der lebendigen Kraft nur um ein hinzutretendes Glied unterscheidet. Die Substitution, welche zu diesem Resultate führt, ist eine wesentlich andere als die in dem erwähnten Aufsatz angewandte. Aber beide haben dies gemein, dafs sie die Bestimmung des Druckes von der übrigen Behandlung der Aufgabe sondern, und auf Gleichungen führen, welche, indem sie von den ãufseren Kräften unabhängig sind, Bewegungen allgemeinstẹr Natur darstellen, deren eine Flüssigkeit fähig ist; endlich, dafs die angewandten neuen abhängigen Variabeln, Canstanten gleich gesetzt, Integrale des hinzutretenden Systems gewöhnlicher Differentialgleichungen bilden. Während aber jene Substitution für die stationăre Bewegung auf zwei partielle Differentialgleichungen zweiter Ordnung führte, kommt in dem vorliegenden Falle das Problem suruck auf drei Differentialgleìchungen, von denen zwei von der ersten, eine von der zweilen Ordnung. 
Clebsch, über die Integration der hydrodynamischen Gleichungen.

Die angewandte Substitution knüpft an die gewöhnliche Methode an, die hydrodynamischen Gleichungen zu behandeln. In der That macht man gewöhnlich die Annahme, dafs der Ausdruck

$$
u d x+v d y+w d z
$$

ein vollständiges Differential sein soll. Aber wie auch die $u, v, w$ beschaffen sein mögen, stets läfst dieser Ausdruck sich auf ein zweigliedriges Differential zurückführen, d. h. auf die Form

$$
d \varphi+m d \psi
$$

woraus sich die Gleichungen ergeben:

$$
\begin{aligned}
\boldsymbol{u} & =\frac{\partial \varphi}{\partial x}+m \frac{\partial \psi}{\partial x}, \\
\boldsymbol{v} & =\frac{\partial \varphi}{\partial y}+m \frac{\partial \psi}{\partial y}, \\
\boldsymbol{w} & =\frac{\partial \varphi}{\partial z}+m \frac{\partial \psi}{\partial z},
\end{aligned}
$$

welches eben die angewandten Substitutionen sind. Ich bemerke, dafs dieselben in einer gewissen Beziehung zu der Betrachtung der von Helmholtz (dieses Journal, Bd 55, p. 25) in die Theorie eingeführten Wirbelbewegungen stehen. Die Geschwindigkeiten sondern sich hier in einen Theil, der durch entsprechende Differentialquotienten einer Function dargestellt wird, und in einen zweiten, der diese Darstellung schlechterdings nicht zuläfst. Jene Wirbelbewegungen nun hängen allein von diesem zweiten Theil $a b, d$. $h$. von den Functionen $m, \psi$. Denn wenn man nach den dort (Form. 2) gegebenen Gleichungen die Rotationsgeschwindigkeiten eines Flüssigkeitstheilchens bildet, so hat man:

$$
\begin{aligned}
& 2 \xi=\frac{\partial m}{\partial z} \frac{\partial \psi}{\partial y}-\frac{\partial m}{\partial y} \frac{\partial \psi}{\partial z} \\
& 2 \eta=\frac{\partial m}{\partial x} \frac{\partial \psi}{\partial z}-\frac{\partial m}{\partial z} \frac{\partial \psi}{\partial x} \\
& 2 \zeta=\frac{\partial m}{\partial y} \frac{\partial \psi}{\partial x}-\frac{\partial m}{\partial x} \frac{\partial \psi}{\partial y}
\end{aligned}
$$

wo die Function $\varphi$ ganz verschwunden ist. *)

*) Beiläufig ergiebt sich hieraus das Verfahren, um, wenn $u, v, w$ irgend gegebene Functionen sind, dem Ausdruck $u d x+v d y+w d z$ die Gestalt $d \varphi+m d \psi$ zu geben. Es sind $m, \psi$, wie aus dem Pfaffschen Problem sonst bekannt, nach den obigen Gleichungen Integrale der Gleichungen:

$$
d x: d y: d z=\frac{\partial v}{\partial z}-\frac{\partial w}{\partial y}: \frac{\partial w}{\partial x}-\frac{\partial u}{\partial z}: \frac{\partial u}{\partial y}-\frac{\partial v}{\partial x} .
$$


Ich wende mich zunächst zu einem allgemeinern System von Gleichungen, welches analoge Eigenschaften mit dem System der Hydrodynamik aufweist.

\$. 2.

Es sei das System von Gleichungen gegeben:

$$
\text { (1.) }\left\{\begin{array}{l}
\frac{\partial \boldsymbol{V}}{\partial x}=\frac{\partial u}{\partial t}+u \frac{\partial u}{\partial x}+u_{1} \frac{\partial u}{\partial x_{1}}+\cdots u_{2 n} \frac{\partial u}{\partial x_{2 n}} \\
\frac{\partial \boldsymbol{V}}{\partial x_{1}}=\frac{\partial u_{1}}{\partial t}+u \frac{\partial u_{1}}{\partial x}+u_{1} \frac{\partial u_{1}}{\partial x_{1}}+\cdots u_{2 n} \frac{\partial u_{1}}{\partial x_{2 n}} \\
\cdot \cdot \cdot \cdot \cdot \cdot \cdot \cdot \cdot \cdot \cdot \cdot \cdot \cdot \cdot \cdot \cdot \cdot \cdot \cdot \cdot \cdot \cdot \cdot \cdot \cdot \frac{\partial u_{2 n}}{\partial x}+u_{1} \frac{\partial u_{2}}{\partial x_{1}}+\cdots u_{2 n} \frac{\partial u_{2 n}}{\partial x_{2 n}} \\
\frac{\partial V}{\partial x_{2 n}}=\frac{\partial u_{2 n}}{\partial t}+u \\
\text { (2.) } \frac{\partial u}{\partial x}+\frac{\partial u_{1}}{\partial x_{1}}+\cdots \frac{\partial u_{2 n}}{\partial x_{2 n}}=0
\end{array}\right.
$$

denen sich die gewöhnlichen Differentialgleichungen anschliefsen sollen:

$$
\text { (3.) } \frac{d x}{d t}=u, \frac{d x_{1}}{d t}=u_{1}, \ldots \frac{d x_{2 n}}{d t}=u_{2 n} \text {. }
$$

Die Gleichungen (1.) lassen sich in einer symbolischen Form zusammenfassen; nämlich, wenn unter dem Zeichen $\delta$ nur die $x$, nicht aber $t$ als

Da der Multiplicator der Gleichungen 1 ist, so kann man aus einem Integral $\psi$ das zweite $m$ durch das Princip des letzten Multiplicators finden. Alsdann aber ist wirklich

$$
\frac{\partial v}{\partial z}-\frac{\partial w}{\partial y}=\frac{\partial m}{\partial z} \frac{\partial \psi}{\partial y}-\frac{\partial \psi}{\partial z} \frac{\partial m}{\partial y} \text { etc. }
$$

und $\varphi$ wird daher wirklich ein vollständiges Differential. Aber $\varphi$ genügt der Differentialgleichung

$$
\begin{gathered}
\left(\frac{\partial v}{\partial z}-\frac{\partial w}{\partial y}\right) \frac{\partial \varphi}{\partial x}+\left(\frac{\partial w}{\partial x}-\frac{\partial u}{\partial z}\right) \frac{\partial \varphi}{\partial y}+\left(\frac{\partial u}{\partial y}-\frac{\partial v}{\partial x}\right) \frac{\partial \varphi}{\partial z} \\
=u\left(\frac{\partial v}{\partial z}-\frac{\partial w}{\partial y}\right)+v\left(\frac{\partial w}{\partial x}-\frac{\partial u}{\partial z}\right)+v\left(\frac{\partial u}{\partial y}-\frac{\partial v}{\partial z}\right) .
\end{gathered}
$$

Führt man hier stalt $x, y, z$ als neue Variable $m, \psi, \vartheta$ ein, so erhält man durch Integration :

$$
\varphi=\int \frac{u\left(\frac{\partial v}{\partial z}-\frac{\partial w}{\partial y}\right)+v\left(\frac{\partial w}{\partial x}-\frac{\partial u}{\partial z}\right)+w\left(\frac{\partial u}{\partial y}-\frac{\partial v}{\partial x}\right)}{\frac{\partial \vartheta}{\partial x}\left(\frac{\partial v}{\partial z}-\frac{\partial w}{\partial y}\right)+\frac{\partial \vartheta}{\partial y}\left(\frac{\partial w}{\partial x}-\frac{\partial u}{\partial z}\right)+\frac{\partial \vartheta}{\partial z}\left(\frac{\partial u}{\partial y}-\frac{\partial v}{\partial x}\right)} d \vartheta
$$

(vgl. Jacobi, Math. W. Bd. I. p. 144). 
veränderlich betrachtet werden:

oder auch, wenn man

$$
\text { (4.) } \quad \delta V=\Sigma_{k} \delta x_{k}\left(\frac{\partial u_{k}}{\partial t}+u \frac{\partial u_{k}}{\partial x}+\cdots u_{2 n} \frac{\partial u_{k}}{\partial x_{2 n}}\right) \text {; }
$$

$$
\mathbf{2 T}=\boldsymbol{u}^{2}+\boldsymbol{u}_{1}^{2}+\cdots \boldsymbol{u}_{2 n}^{2}
$$

setzt, in der folgenden:

(6.) $\quad \delta(\boldsymbol{V}-\boldsymbol{T})=\Sigma_{k} \frac{\partial u_{k}}{\partial t} \delta x_{k}+\frac{1}{2} \Sigma_{i} \Sigma_{k}\left(\frac{\partial u_{k}}{\partial x_{i}}-\frac{\partial u_{i}}{\partial x_{k}}\right)\left(u_{i} \delta x_{k}-u_{k} \delta x_{i}\right)$.

Bemerken wir nun, dafs man dem Ausdruck:

$$
u \delta x+u_{1} \delta x_{1}+\cdots u_{2 n} \delta x_{2 n}
$$

immer die $n$ nachstehende Gestalt geben kann:

$$
\delta \varphi+m_{1} \delta \varphi_{1}+m_{2} \delta \varphi_{2}+\cdots m_{n} \delta \varphi_{n},
$$

so werden wir darauf 'geführt, für die $u$ die Substitutionen zu machen:

$$
\boldsymbol{u}_{k}=\frac{\partial \varphi}{\partial x_{k}}+\boldsymbol{m}_{1} \frac{\partial \varphi_{1}}{\partial x_{k}}+m_{2} \frac{\partial \varphi_{2}}{\partial x_{k}}+\cdots m_{n} \frac{\partial \varphi_{n}}{\partial x_{k}} .
$$

Mit Hülfe dieser Substitutionen stellen sich zunächst die Ausdrücke $\frac{\partial u_{k}}{\partial x_{i}}-\frac{\partial u_{i}}{\partial x_{k}}$ als Summen von Determinanten dar, nämlich,

$$
\frac{\partial u_{k}}{\partial x_{i}}-\frac{\partial u_{i}}{\partial x_{k}}=\Sigma_{r}\left(\frac{\partial m_{r}}{\partial x_{i}} \frac{\partial \varphi_{r}}{\partial x_{k}}-\frac{\partial m_{r}}{\partial x_{k}} \frac{\partial \varphi_{r}}{\partial x_{i}}\right) \text {. }
$$

Wenn man aber diesen Ausdruck mit der Determinante $u_{i} \delta x_{k}-u_{k} \delta x_{i}$ multiplicirt, und sodann nach $k, i$ die Summe bildet, so erhält man nach bekannten Sätzen:

$$
\begin{aligned}
& \frac{1}{2} \Sigma_{i} \Sigma_{k}\left(\frac{\partial u_{k}}{\partial x_{i}}-\frac{\partial u_{i}}{\partial x_{k}}\right)\left(u_{i} \delta x_{k}-u_{k} \delta x_{i}\right) \\
& =\Sigma_{r}\left|\begin{array}{l}
u \frac{\partial m_{r}}{\partial x}+u_{1} \frac{\partial m_{r}}{\partial x_{1}}+\cdots, \frac{\partial m_{r}}{\partial x} \delta x+\frac{\partial m_{r}}{\partial x_{1}} \delta x_{1}+\cdots \\
u \frac{\partial p_{r}}{\partial x}+u_{1} \frac{\partial \varphi_{r}}{\partial x_{1}}+\cdots, \frac{\partial \varphi_{r}}{\partial x} \delta x+\frac{\partial \varphi_{r}}{\partial x_{1}} \delta x_{1}+\cdots
\end{array}\right| .
\end{aligned}
$$

Hiefür kann man mit Berücksichtigung der. Gleichungen (3.) die kứrzere Bezeichnung anwenden:

$$
\Sigma_{r}\left\{\left(\frac{d m_{r}}{d t}-\frac{\partial m_{r}}{\partial t}\right) \delta \varphi_{r}-\left(\frac{d \varphi_{r}}{d t}-\frac{\partial \varphi_{r}}{\partial t}\right) \delta m_{r}\right\} .
$$

Setzen wir dies nun in die Gleichung (6.) ein, so vereinigt sich die Summe

$$
\Sigma_{k} \frac{\partial u_{k}}{\partial t} \delta x_{k}=\delta \frac{\partial \varphi}{\partial t}+\Sigma_{r}\left(m_{r} \delta \frac{\partial \varphi_{r}}{\partial t}+\frac{\partial m_{r}}{\partial t} \delta \varphi_{r}\right)
$$


Clebsch, über die Integration der hydrodynamischen Gleichungen.

mit dem Theile

$$
\Sigma_{r}\left(\frac{\partial \varphi_{r}}{\partial t} \delta m_{r}-\frac{\partial m_{r}}{\partial t} \delta \varphi_{r}\right)
$$

der obigen Summe, zı der vollständigen Variation des Ausdrucks

$$
\frac{\partial \varphi}{\partial t}+\Sigma_{r} m_{r} \frac{\partial \varphi_{r}}{\partial t}
$$

und die Gleichung (6.) nimmt demnach folgende Gestalt an:

$$
\delta\left\{V-T-\frac{\partial \varphi}{\partial t}-\Sigma_{r} m_{r} \frac{\partial \varphi_{r}}{\partial t}\right\}=\Sigma_{r}\left(\frac{d m_{r}}{d t} \delta \varphi_{r}-\frac{d \varphi_{r}}{d t} \delta m_{r}\right) .
$$

Diese Gleichung aber enthält auf der rechten Seite nur $2 n$ Variationen $\delta m, \delta \varphi$, während in der linken Seite $2 n+1$ Variable $x$ variirt werden. Es mufs also der Ausdruck, welcher links variirt ist, dine willkŭrliche Function der $2 n+1$ Argumente $\varphi, m, t$ sein; und indem wir die Bezeichnungen $\frac{d m}{d t}, \frac{d \varphi}{d t}$ wieder auflösen, können wir folgendes Theorem aussprechen:

\section{S a tz 1.}

Die Gleichungen (1.), (2.) lassen sich durch das System ersetzen:

wo

$$
\text { (9.) }\left\{\begin{aligned}
\frac{\partial m_{r}}{\partial t}+u \frac{\partial m_{r}}{\partial x}+u_{1} \frac{\partial m_{r}}{\partial x_{1}}+\cdots & =\Pi^{\prime} \varphi_{r}, \\
\frac{\partial \varphi_{r}}{\partial t}+u \frac{\partial \varphi_{r}}{\partial x}+u_{1} \frac{\partial \varphi_{r}}{\partial x_{1}}+\cdots & =-\Pi^{\prime} m_{r}, \\
\frac{\partial u}{\partial x}+\frac{\partial u_{1}}{\partial x_{1}}+\cdots \frac{\partial u_{2 n}}{\partial x_{2 n}} & =0
\end{aligned}\right.
$$

$$
\boldsymbol{u}_{k}=\frac{\partial \varphi}{\partial x_{k}}+m_{1} \frac{\partial \varphi_{1}}{\partial x_{k}}+m_{2} \frac{\partial \varphi_{2}}{\partial x_{k}}+\cdots m_{n} \frac{\partial \varphi_{n}}{\partial x_{k}}
$$

und wo $I$ eine willkürliche Function von $t, \varphi_{1}, \ldots \varphi_{n}, m_{1}, \ldots m_{n}$ bedeutet.

Dieses System enthäl $2 n$ Gleichungen der ersten, eine der zweiten Ordnung. Nachdem es integrirt, ergeben sich die $u$ von selbst aus obiger Gleichung; $\boldsymbol{V}$ ist bestimmt durch die Gleichung:

$$
\text { (10.) } V=\left(\frac{\partial \varphi}{\partial t}+\Sigma_{r} m_{r} \frac{\partial \varphi_{r}}{\partial t}\right)+\frac{1}{2} \Sigma_{k}\left(\frac{\partial \varphi}{\partial x_{k}}+\Sigma_{r} m_{r} \frac{\partial \varphi_{r}}{\partial x_{k}}\right)^{2}+I \text {. }
$$

Die Gleichungen (3.) endlich kommen zurück auf das Syslem:

$$
\text { (11.) } \frac{d \varphi_{r}}{d t}=-\Pi^{\prime} m_{r}, \quad \frac{d m_{r}}{d t}=\Pi^{\prime} \varphi_{r} \text {. }
$$

Das fehlende Integral des Systems (3.), welches eine Gleichung mehr 
als das vorliegende enthält, giebt das Princip des letzten Multiplicators.

Diesem Theoreme kann man das folgende hinzufügen, welches sich ohne Weiteres verificiren läfst:

S a tz 2.

Die Gleichungen (9.) machen das Integral

$$
\int^{2 n+2} V d x d x_{1} \ldots d x_{2 n} d t
$$

zu einem Maximum oder Minimum, $V$ durch die Gleichung (10.) ausgedrückt gedacht.

Diese Gleichungen enthalten eine willkürliche Function $\Pi$. Inzwischen, da man annehmen darf, dafs die Integrale eines Systems von $2 n$ Gleichungen der ersten und einer Gleichung der zweiten Ordnung, an sich ebenso viel Willkürlichkeiten enthalten müssen, als die Integrale eines Systems von $2 n+2$ Gleichungen erster Ordnung; so scheint es dafs die Gleichungen (9.), in welche aufserdem noch $\Pi$ eingeht, mehr Willkürliches mit sich fübren, als der Natur der Aufgabe nach zulässig ist. Dieser Ueberschufs von Willkürlichem darf daher auf die abhängigen Functionen des ursprūnglichen Problems keinen Einflufs ausüben; er mufs aus den Ausdrüeken von $V, u, u_{1}, \ldots u_{2 n}$ verschwinden. Ich werde nun in der That zeigen,

dafs es, ohne die Allgemeinheit der Werthe van $V, u, u_{1}, \ldots u_{2 n} \approx u$ beeinträchtigen, erlaubt ist, die Function $\Pi$ gleich Null zu setzen.

\$. 3.

Die Gleichungen (11.) baben die canonische Form, welche bekanntlich es erlaubt, den Integralen dieser Gleichungen eine entsprechende Form zu geben, und dieselben auszudrücken durch die vollständige Lösung einer partiellen Differentialgleichung. In der That, man kann stets eine Function $(\boldsymbol{W})$ von $t, \varphi_{1}, \varphi_{2}, \ldots \varphi_{n}$ und von $n$ Constanten $a_{1}, a_{2}, \ldots a_{n}$ so bestimmen, dafs

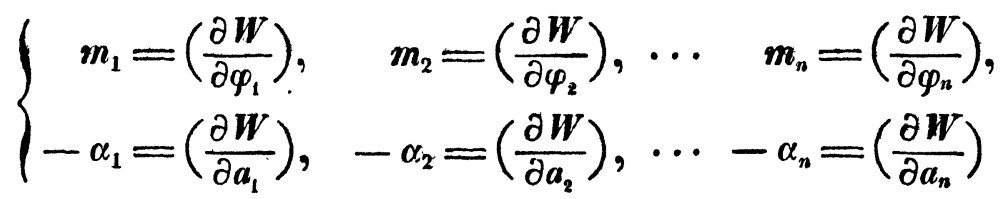

die Integrale der Gleichungen (11.) sind, wăhrend die $\alpha$ neue Constanten bedeuten; und man hat ferner

(13.) $\left(\frac{\partial W}{\partial t}\right)=\Pi$, 
woraus die für $\boldsymbol{W}$ bestehende partielle Differentialgleichung hervorgeht, wenn man aus $\Pi$ die $m$ mit Hülfe der ersten Gleichungen (12.) eliminirt.

Man kann nun offenbar statt der Fanction $\Pi$ die eine gleiche Willkürlichkeit enthaltende Function $(\boldsymbol{W})$ in die Rechnung einführen; wo nur, sobald man von dén gewöhnlichen Differentialgleichungen zu den partiellen übergeht, die $a, \alpha$ nicht mehr als Constante, sondern als Functionen von $t, x, x_{1}, \ldots x_{2 n}$ zu betrachten sind. Und zugleich kann man auch statt der $m, \varphi$ sich diese Functionen $a, \alpha$ in die Gleichungen als abhängige Variable eingeführt denken; wo denn auch in $(\boldsymbol{W})$ die $\varphi$ durch diese Functionen zu ersetzen sind. Sehen wir, wie sich die Functionen $\boldsymbol{V}, \boldsymbol{u}$ durch diese neuen abhängigen Variabeln ausdrücken.

Wenn man die Gleichungen (12.) in die Ausdrücke der $\boldsymbol{u}$ einführt, so gehen dieselben zunächst über in:

$$
\boldsymbol{u}_{k}=\frac{\partial \varphi}{\partial x_{k}}+\left(\frac{\partial \boldsymbol{W}}{\partial \varphi_{1}}\right) \frac{\partial \varphi_{1}}{\partial x_{k}}+\left(\frac{\partial \boldsymbol{W}}{\partial \varphi_{2}}\right) \frac{\partial \varphi_{2}}{\partial x_{k}}+\cdots\left(\frac{\partial \boldsymbol{W}}{\partial \varphi_{n}}\right) \frac{\partial \varphi_{n}}{\partial x_{k}} .
$$

Bezeichnen wir aber durch $\boldsymbol{W}$ die Function $(\boldsymbol{W})$, wenn wir sie als Function der $t, x, x_{1}, \ldots x_{2 n}$ betrachten, so ist offenbar:

$$
\frac{\partial \boldsymbol{W}}{\partial x_{k}}=\left(\frac{\partial \boldsymbol{W}}{\partial \varphi_{1}}\right) \frac{\partial \varphi_{1}}{\partial x_{k}}+\left(\frac{\partial \boldsymbol{W}}{\partial \varphi_{2}}\right) \frac{\partial \varphi_{2}}{\partial x_{k}}+\cdots+\left(\frac{\partial \boldsymbol{W}}{\partial a_{1}}\right) \frac{\partial a_{1}}{\partial x_{k}}+\left(\frac{\partial W}{\partial a_{2}}\right) \frac{\partial a_{2}}{\partial x_{k}}+\cdots
$$

und man kann also, wieder mit Hülfe der Gleichungen (12.), den obigen Ausdruck von $\boldsymbol{u}$ auch durch den folgenden ersetzen:

$$
\text { (14.) } \quad u_{k}=\frac{\partial \cdot \varphi+W}{\partial x_{k}}+\alpha_{1} \frac{\partial a_{1}}{\partial x_{k}}+\alpha_{2} \frac{\partial a_{2}}{\partial x_{k}}+\cdots \alpha_{n} \frac{\partial a_{n}}{\partial x_{k}} \text {. }
$$

Bemerken wir noch, dafs auch

$$
\frac{\partial W}{\partial t}=\left(\frac{\partial W}{\partial t}\right)+\left(\frac{\partial W}{\partial \varphi_{1}}\right) \frac{\partial \varphi_{1}}{\partial t}+\left(\frac{\partial W}{\partial \varphi_{2}}\right) \frac{\partial \varphi_{2}}{\partial t}+\cdots+\left(\frac{\partial W}{\partial u_{1}}\right) \frac{\partial a_{1}}{\partial t}+\left(\frac{\partial W}{\partial a_{2}}\right) \frac{\partial a_{2}}{\partial t}+\cdots,
$$

so geht der Ausdruck

$$
\boldsymbol{\Pi}+\frac{\partial \varphi}{\partial t}+\Sigma_{r} m_{r} \frac{\partial \varphi_{r}}{\partial t}==^{\prime}\left(\frac{\partial \boldsymbol{W}}{\partial t}\right)+\frac{\partial \varphi}{\partial t}+\Sigma_{r}\left(\frac{\partial \boldsymbol{W}}{\partial \varphi_{r}}\right) \frac{\partial \varphi_{r}}{\partial t}
$$

in den folgenden über:

$$
\frac{\partial \cdot \varphi+W}{\partial t}+\alpha_{1} \frac{\partial a_{1}}{\partial t}+\alpha_{2} \frac{\partial a_{2}}{\partial t}+\cdots \alpha_{n} \frac{\partial a_{n}}{\partial t} .
$$

Daher nimmt die Gleichung (10.) unmittelbar die Gestalt an:

(15.) $\boldsymbol{V}=$

$$
\frac{\partial \cdot \varphi+W}{\partial t}+\alpha_{1} \frac{\partial a_{1}}{\partial t}+\alpha_{2} \frac{\partial a_{2}}{\partial t}+\cdots+\frac{1}{2} \Sigma_{k}\left(\frac{\partial \cdot \varphi+W}{\partial x_{k}}+\alpha_{1} \frac{\partial a_{1}}{\partial x_{k}}+\alpha_{2} \frac{\partial a_{2}}{\partial x_{k}}+\cdots\right)^{2}
$$


8 Clebsch, über die Integration der hydrodynamischen Gleichungen.

Vergleichen wir jetzt die Gleichungen (14.), (15.) mit den Gleichungen (7.), (10.). Man sieht sogleich, dafs nur an die Stelle von $\varphi$ die Function $\varphi+W$ getreten ist; an die Stelle der $m$ aber die $\alpha$, an die Stelle der $\varphi$ die $a$; endlich, dafs die Function $\Pi$ verschwunden ist. Da nun ferner den Gleichungen (11.) offenbar die folgenden entsprechen:

$$
\text { (16.) } \quad \frac{d a_{r}}{d t}=0, \quad \frac{d \alpha_{r}}{d t}=0,
$$

welche, aufgelöst, Gleichungen ergeben, die den Gleichungen (9.) vollständig ähnlich sind; so sieht man ein, dafs das jetzt erlangte reducirte Problem sich von dem in dem Satz 1. enthaltenen nur dadurch unterscheidet, dafs die Function $\Pi$ gleich Null gesetzt ist, und dafs an die Stelle der Buchstaben $\varphi$, $m$ andere getreten sind. Aber zugleich werden die gewöhnlichen Differentialgleichungen integrabel; und indem wir also zu den frühern Bezeichnungen zurückkehren, können wir folgendes Theorem aufstellen:

S a t z 3.

Die Gleichungen (1.), (2.) können ersetzt werden durch das System

$$
\text { (17.) }\left\{\begin{array}{c}
\frac{\partial m_{r}}{\partial t}+u \frac{\partial m_{r}}{\partial x}+u_{1} \frac{\partial m_{r}}{\partial x_{1}}+\cdots u_{2 n} \frac{\partial m_{r}}{\partial x_{2 n}}=0 \\
\frac{\partial \varphi_{r}}{\partial t}+u \frac{\partial \varphi_{r}}{\partial x}+u_{1} \frac{\partial \varphi_{r}}{\partial x_{1}}+\cdots u_{2 n} \frac{\partial \varphi_{r}}{\partial x_{2 n}}=0 \\
\frac{\partial u}{\partial x}+\frac{\partial u_{1}}{\partial x_{1}}+\cdots \frac{\partial u_{2 n}}{\partial x_{2 n}}=0
\end{array}\right.
$$

wo

$$
\boldsymbol{u}_{k}=\frac{\partial \varphi}{\partial x_{k}}+m_{1} \frac{\partial \varphi_{1}}{\partial x_{k}}+m_{2} \frac{\partial \varphi_{2}}{\partial x_{k}}+\cdots m_{n} \frac{\partial \varphi_{n}}{\partial x_{k}} .
$$

Von diesen Gleichungen sind zwei von der ersten, eine von der zweiten Ordnung. Nachdem sie integrirt sind, ergeben sich die $u$ aus der ohigen Formel, $V$ aber aus der Gleichung:

$$
\text { (18.) } \quad \begin{aligned}
\quad & \boldsymbol{V}=\frac{\partial \varphi}{\partial t}+m_{1} \frac{\partial \varphi_{1}}{\partial t}+m_{2} \frac{\partial \varphi_{2}}{\partial t}+\cdots m_{n} \frac{\partial \varphi_{n}}{\partial t} \\
& +\frac{1}{2} \Sigma_{k}\left\{\frac{\partial \varphi}{\partial x_{k}}+m_{1} \frac{\partial \varphi_{1}}{\partial x_{k}}+m_{2} \frac{\partial \varphi_{2}}{\partial x_{k}}+\cdots m_{n} \frac{\partial \varphi_{n}}{\partial x_{k}}\right\}^{2} ;
\end{aligned}
$$

und die Gleichungen (3.) haben $2 u$ Integralen die Gleichungen:

$$
\text { (19.) } m_{r}=\text { Const., } \quad \varphi_{r}=\text { Const., }
$$

zu denen ein letzles Integral trill, welches aus den Gleichungen (3.) und (19.) mit Hülfe des letzlen Muliplicators gewonnen wird. 
Hierdurch also ist das vorgelegte System zurückgeführt auf ein anderes, welches nicht mehr Willkürlichkeiten in seiner allgemeinen Lösung enthält als jenes; und welches die Eigenschaft hat, die Integrale der hinzutretenden gewöhnlichen Differentialgleichungen ohne Weiteres zu ergeben. - Man fügt leicht das folgende Theorem hinzu:

S a tz 4 .

Die Gleichungen (17.) machen das Integral

$$
\int^{(2 n+2)} V d x d x_{1} \ldots d x_{2 n} d t
$$

zu. einem Maximum oder Minimum, wo V durch die Gleichung (18.) ausgedrückt zu denken ist.

\section{\$. 4 .}

Es bleibt nichts übrig, als die erlangten Resultate nunmehr für den Fall der Hydrodynamik auszusprechen, für welchen $n=1$ ist. 'Sei $U$ die Kräftefunction, $p$ der Druck, $q$ die Dichtigkeit, so hat man demnach folgenden Satz:

\section{Die Gleichungen}

$$
\left\{\begin{aligned}
& \frac{\partial}{\partial x}\left(U-\frac{p}{q}\right)=\frac{\partial u}{\partial t}+u \frac{\partial u}{\partial x}+v \frac{\partial u}{\partial y}+w \frac{\partial u}{\partial z} \\
& \frac{\partial}{\partial y}\left(U-\frac{p}{q}\right)=\frac{\partial v}{\partial t}+u \frac{\partial v}{\partial x}+v \frac{\partial v}{\partial y}+w \frac{\partial v}{\partial z} \\
& \frac{\partial}{\partial z}\left(U-\frac{p}{q}\right)=\frac{\partial w}{\partial t}+u \frac{\partial w}{\partial x}+v \frac{\partial w}{\partial y}+w \frac{\partial w}{\partial z} \\
& \frac{\partial u}{\partial x}+\frac{\partial v}{\partial y}+\frac{\partial w}{\partial z}=0
\end{aligned}\right.
$$

lassen sich auf die Aufgabe zurückführen, das Integral

$$
\iiint \int\left(U-\frac{p}{q}\right) d t d x d y d z
$$

zu einem Maximum oder Minimum zu machen, wo

$$
\text { (21.) } U-\frac{p}{q}=\frac{\partial \varphi}{\partial t}+m \frac{\partial \psi}{\partial t}+\frac{u^{2}+v^{2}+w^{2}}{2}
$$

zu setzen ist, und für die $u, v, w$ die Ausdrücke gelten: Journal für Mathematik Bd. LVI. Heft 1. 
10 Clebsch, ̈̈ber die Integration der hydrodynamischen Gleichungen.

$$
\text { (22.) }\left\{\begin{array}{l}
\boldsymbol{u}=\frac{\partial \varphi}{\partial x}+m \frac{\partial \psi}{\partial x} \\
\boldsymbol{v}=\frac{\partial \varphi}{\partial y}+m \frac{\partial \psi}{\partial y} \\
\boldsymbol{w}=\frac{\partial \varphi}{\partial z}+m \frac{\partial \psi}{\partial z}
\end{array}\right.
$$

Die Integrale der Gleichungen

$$
\frac{d x}{d t}=u, \quad \frac{d y}{d t}=v, \quad \frac{d z}{d t}=w
$$

werden dann:

$$
\text { (23.) } \quad m=\text { Const. }, \quad \psi=\text { Const. }
$$

und ein drittes, welches die Theorie des letzten Multiplicators ergiebt. Die Gleichungen, auf welche die Aufgabe zurückkommt, werden hiernach:

$$
\begin{aligned}
& \mathbf{0}=\frac{\partial m}{\partial t}+\left(\frac{\partial m}{\partial x} \frac{\partial \varphi}{\partial x}+\frac{\partial m}{\partial y} \frac{\partial \varphi}{\partial y}+\frac{\partial m}{\partial z} \frac{\partial \varphi}{\partial z}\right)+m\left(\frac{\partial m}{\partial x} \frac{\partial \psi}{\partial x}+\frac{\partial m}{\partial y} \frac{\partial \psi}{\partial y}+\frac{\partial m}{\partial z} \frac{\partial \psi}{\partial z}\right), \\
& 0=\frac{\partial \psi}{\partial t}+\left(\frac{\partial \psi}{\partial x} \frac{\partial \varphi}{\partial x}+\frac{\partial \psi}{\partial y} \frac{\partial \varphi}{\partial y}+\frac{\partial \psi}{\partial z} \frac{\partial \varphi}{\partial z}\right)+m\left(\frac{\partial \psi}{\partial x} \frac{\partial \psi}{\partial x}+\frac{\partial \psi}{\partial y} \frac{\partial \psi}{\partial y}+\frac{\partial \psi}{\partial z} \frac{\partial \psi}{\partial z}\right), \\
& 0=\frac{\partial}{\partial x}\left(\frac{\partial \varphi}{\partial x}+m \frac{\partial \psi}{\partial x}\right)+\frac{\partial}{\partial y}\left(\frac{\partial \varphi}{\partial y}+m \frac{\partial \psi}{\partial y}\right)+\frac{\partial}{\partial z}\left(\frac{\partial \varphi}{\partial z}+m \frac{\partial \psi}{\partial z}\right) .
\end{aligned}
$$

Es ist sehr leicht von diesen Gleichungen, für den stationären Zustand, zu denjenigen überzugehen, welche ich an dem angeführten Orte entwickelt habe; indem man nur bemerkt, wie aus der im Anfange des $\mathbf{\$} .3$ aufgestellten Betrachtung sich ergiebt, dafs man nur eines der $m, \psi$ von $t$ vollkommen frei annehmen darf, während das andere von der Form $t \boldsymbol{f}+\boldsymbol{F}$ ist.

Ich bemerke nur noch, dafş die Gleichung (21.) die der lebendigen Kraft ist; in einer sehr bekannten Gestalt, wenn man, zu der gewöhnlichen Annahme zurückkehrend, $m$ verschwinden läfst.

Berlin, den 1. März 1858. 